\title{
Comment on "State of the art in magnetic resonance imaging of hepatocellular carcinoma": the role of DWI
}

\author{
Vincenza Granata1, Roberta Fusco ${ }^{1}$, Salvatore Filice1, Paola Incollingo ${ }^{2,3}$, Andrea Belli³, \\ Francesco $\mathrm{IzzO}^{3}$, Antonella Petrillo ${ }^{1}$

\begin{abstract}
${ }^{1}$ Division of Radiology, “Istituto Nazionale Tumori IRCCS Fondazione Pascale - IRCCS di Napoli”, Naples, Italy
${ }^{2}$ Operative Unit of General Surgery and Kidney Transplantation, Advanced Biomedical Science Department University

Federico II of Naples, Naples, Italy

${ }^{3}$ Division of Hepatobiliary Surgical Oncology, "Istituto Nazionale Tumori IRCCS Fondazione Pascale - IRCCS di Napoli”, Naples, Italy
\end{abstract}

Radiol Oncol 2019; 53(3): 369-370.

Received 6 February 2019

Correspondence to: Roberta Fusco, Division of Radiology, "Istituto Nazionale Tumori IRCCS Fondazione Pascale - IRCCS di Napoli”, Naples, Italy. E-mail: r.fusco@istitutotumori.na.it

Disclosure: No potential conflicts of interest were disclosed.

We read with interest the article from Dr Horvat and colleagues in Radiology and Oncology in which they assessed the State of the art in magnetic resonance imaging (MRI) of hepatocellular carcinoma (HCC). ${ }^{1}$ The main strength is due to the good update on the role of this technique for HCC. We congratulate the authors on their accuracy in data presentation. Nevertheless, we would like to point out several features that the Radiology and Oncology readers should know about the Diffusion Weighted Imaging (DWI) and the possibility to use quantitative parameters extracted by DWI to characterize HCC, data not assessed by authors. ${ }^{1}$ DWI has been applied to liver imaging as an excellent tool for detection and characterization of focal liver lesions. The assessment of DW images can be done qualitatively and quantitatively, through the apparent diffusion coefficient (ADC) map. Le Bihan et al. as a first described the intravoxel incoherent motion (IVIM). IVIM data can be assessed qualitatively and quantitatively. IVIM data enable improved detection and characterization of HCC. ${ }^{2}$ Also, traditionally DWI approach to analyze data is founded on the hypothesis that water molecules diffuse within a voxel following a single direction with a Gaussian behavior without any restriction. However, water molecules within biologic tissues exhibits a non-Gaussian phenomena proposed by Jensen in 2005 called Diffusion Kurtosis Imaging
(DKI). ${ }^{3}$ DKI is more accurate than traditional ADC in tumor assessment. ${ }^{3}$ The role of DWI and functional parameters extracted by DWI in HCC patient has been evaluated by different studies, showed that the DWI could be used as a helpful diagnostic tool for HCC in patients with chronic liver disease, since DWI can accurately detect HCC in patients with chronic liver disease regardless of the lesion size. The major limits of DWI are the different parameters used in DWI sequences, which may affect the results of ADC calculation. Several researches have evaluated the relationship between functional data by DWI and histological grade of HCC. Granata et al. ${ }^{4}$ found that DWI could be used to predict the histological grade of HCC, showing a good correlation between ADC and grading, between perfusion fraction ( $\mathrm{fp}$ ) and grading, and between tissue pure diffusivity (Dt) and grading. The mayor limit of DWI and IVIM parameters to discriminate the histological grade of HCC is depending on the fitting methods used to obtained functional parameters.

Goshima et al compared DKI with conventional DWI for assessing the response to treatment in hypervascular HCC. ${ }^{5}$ The sensitivity, specificity, and AUC of the ROC curve for the assessment of HCC viability were greater using MK than using ADC. ${ }^{5}$

In conclusion, DWI should be an integral part of study protocol for HCC patients, considering the 
great advantages due to DWI and DWI-based approaches in detection and characterization of HCC.

\section{Acknowledgement}

The authors are grateful to Alessandra Trocino, librarian at the National Cancer Institute of Naples, Italy. Additionally, authors are grateful to Assunta Zazzaro for the collaboration.

\section{References}

1. Horvat N, Monti S, Oliveira BC, Rocha CCT, Giancipoli RG, Mannelli L. State of the art in magnetic resonance imaging of hepatocellular carcinoma. Radio Oncol 2018; 52: 353-64. doi: 10.2478/raon-2018-0044

2. Le Bihan $\mathrm{D}$, Breton $\mathrm{E}$, Lallemand $\mathrm{D}$, Aubin $\mathrm{ML}$, Vignaud J, Laval-Jeantet $\mathrm{M}$. Separation of diffusion and perfusion in intravoxel incoherent motion MR imaging. Radiology 1988; 168: 497505.

3. Jensen JH, Helpern JA. MRI quantification of non-Gaussian water diffusion by kurtosis analysis. NMR Biomed 2010; 23: 698-710. doi: 10.1002/ nbm.1639

4. Granata V, Fusco R, Filice S, Catalano O, Piccirillo M, Palaia R, et al. The current role and future prospectives of functional parameters by diffusion weighted imaging in the assessment of histologic grade of HCC. Infect Agent Cancer 2018; 3: 23. doi: 10.1186/s13027-018-0194-5

5. Goshima S, Kanematsu M, Noda Y, Kondo H, Watanabe H, Bae KT. Diffusion kurtosis imaging to assess response to treatment in hypervascular hepatocellular carcinoma. AJR Am J Roentgenol 2015; 204: W543-9. doi: 10.2214/ AJR.14.13235 